\title{
Cerebrovascular response to exercise interacts with individual genotype and amyloid-beta deposition to influence response inhibition with aging
}

2 Jacqueline A. Palmer', ${ }^{1}$, Carolyn S. Kaufman ${ }^{3}$, Eric D. Vidoni², Robyn A. Honea ${ }^{2}$, Jeffrey M.

3 Burns, ${ }^{2}$ Sandra A. Billinger ${ }^{1,2 *}$

4

$5 \quad{ }^{1}$ Department of Physical Therapy, Rehabilitation Science, and Athletic Training, School of Health

6 Professions, University of Kansas Medical Center, Kansas City, KS, United States of America

$7 \quad{ }^{2}$ University of Kansas Alzheimer's Disease Center, Fairway, KS, United States of America

$8{ }^{3}$ Department of Molecular \& Integrative Physiology, University of Kansas Medical Center, Kansas

9 City, KS, USA

10

$11 *$ Correspondence:

12 Sandra A. Billinger, PT, PhD, FAHA

13 sbillinger@kumc.edu

14 Keywords: cardiovascular, cognition, executive function, Stroop, Alzheimer's disease, transcranial

15 Doppler

16 Abbreviations: $\mathrm{CVR}=$ cerebrovascular response; $\mathrm{A} \beta=$ amyloid-beta; $\mathrm{APOE} 4=$ Apolipoprotein $\mathrm{E}$

17 allele 4; $\mathrm{AD}=$ Alzheimer's Disease; $\mathrm{MCI}=$ mild cognitive impairment; ANOVA = analysis of

18 variance; $\mathrm{SD}=$ standard deviation 


\section{Highlights}

21 - Neurobiological interactions between CVR, APOE genotype, and A $\beta$ are behaviorally significant.

- Blunted CVR to exercise is associated with impaired response inhibition specifically in APOE4 carriers.

- APOE4 carriers with more robust CVR have higher response inhibition performance, despite having greater $A \beta$ deposition.

- Assessment of multifactorial neurobiological variables offers an early and sensitive biomarker of cognitive behavioral dysfunction with aging. 


\section{Abstract}

31 The etiology of cognitive dysfunction associated with Alzheimer's disease (AD) and dementia is

32 multifactorial. Yet, mechanistic interactions among key neurobiological factors linked to AD

33 pathology are unclear. This study tested the effect of interactions between cerebrovascular function,

34 individual genotype, and structural brain pathology on response inhibition performance, an early and

35 sensitive indicator of cognitive executive dysfunction with aging.

36 We quantified cerebrovascular response (CVR) to moderate-intensity aerobic exercise using

37 transcranial doppler ultrasound and global amyloid-beta $(\mathrm{A} \beta)$ deposition using positron emission

38 tomography in a group of cognitively normal older adults genotyped as APOE4 carriers and

39 noncarriers. We quantified response inhibition during a cognitive Stroop test.

40 Individuals with blunted CVR possessed greater A $\beta$ deposition. There was CVR-by-carrier status-by-

$41 \mathrm{~A} \beta$ interaction on response inhibition. Blunted CVR was associated with impaired response

42 inhibition specifically in carriers. Despite having greater A $\beta$ deposition, carriers with higher CVR

43 demonstrated better response inhibition.

44 Cerebrovascular interactions with individual genotype and structural brain pathology may provide a

45 physiologically-informed target for precision-medicine approaches for early treatment and prevention

46 of cognitive dysfunction with aging. 


\section{Introduction}

Among a growing older adult population, the development of cognitive dysfunction is common but poorly understood. Profound individual differences in aging trajectories allow some older adults to maintain cognitive performance to the end of life while others suffer the debilitating loss of cognitive function associated with Alzheimer's disease (AD) and related dementias (ADRD). The field of aging neurobiology increasingly recognizes cognitive decline with aging as having a mixed pathology and multifactorial etiology (Iturria-Medina et al., 2016, 2017); thus, a trans-disciplinary approach is imperative to enhance our understanding of the diverse and interacting mechanisms leading to ADRD. In the present study, we provide an initial characterization of interactions among key neurobiological factors that have been identified as significant contributors to the development of cognitive dysfunction and AD, including cerebrovascular function (Iadecola, 2004; Xie et al., 2016; Ouellette and Lacoste, 2021), structural brain pathology (Jack et al., 2010; Hampel, 2013), and individual genotype (Heffernan et al., 2016). Further, we investigate the behavioral relevance of these interactions on response inhibition performance in cognitively normal older adults.

Early detection of pathologic disease processes is imperative as a first step towards the development of effective prevention strategies and interventions for ADRD. One of the earliest behavioral manifestations of mild cognitive impairment (MCI) that can progress to AD has been identified as impaired cognitive executive function (Hutchison et al., 2010; Kirova et al., 2015). Specifically, the ability to suppress an undesired default or automatic response in the presence of interfering stimuli, or response inhibition, can distinguish between older adults who are cognitively normal versus MCI (Hutchison et al., 2010). Additionally, cortical activity dysfunction during response inhibition may underpin cognitive interference in motor behavior (Palmer et al., 2021), a common observation in older adults with MCI and early-stage AD (Coelho et al., 2012; Kirova et al., 2015), and older adults at high genetic risk for AD (Whitson et al., 2018). Yet the neurobiologic and physiologic mechanisms that contribute to the early-stage development of impaired response inhibition behavior remain elusive. Such knowledge could be leveraged towards the development of prevention strategies for clinical syndromes in the early stages of MCI, AD and related dementias.

Cerebrovascular pathology is commonly detected in older adults who present with behavioral deficits associated with clinical AD syndrome (Lorius et al., 2015; Wolters Frank J. et al., 2017; Sweeney et al., 2018; Bracko et al., 2021) and is linked to biomarkers of brain pathology, particularly the deposition of amyloid-beta (Zlokovic et al., 2005; Sisante et al., 2019; Solis et al., 2020). The dynamic characterization of cerebral blood flow regulation under conditions of physiologic stress enables the assessment of the cerebrovascular regulatory response to a wide variety of physiological inputs common in daily activities (Ferguson, 2014), including altered perfusion pressure, arterial blood gas, neural activity, and brain metabolism (Smith and Ainslie, 2017). Indeed, cerebrovascular regulation under such conditions of physiologic stress (e.g. sit-to-stand positional changes, aerobic exercise, heat stress, hypoxia) appear to play an important role in maintaining brain metabolism and function with aging (Bundo et al., 2002; Ogoh and Ainslie, 2009a, 2009b; Sato et al., 2011; Ogoh et al., 2013; Steinback and Poulin, 2016), as the effects of repeated transient disruption of blood, glucose, and oxygen supply to brain tissue accumulate over time (Tarumi and Zhang, 2018). Impaired cerebral blood flow can promote ischemic microlesions (Iadecola, 2004), and alter bloodbrain barrier trafficking of amyloid-beta $(\mathrm{A} \beta)$ (Zlokovic et al., 2005), slowing the clearance of $\mathrm{A} \beta$ 90 and promoting its accumulation in the brain.

91 Using transcranial Doppler ultrasound (TCD) to assess cerebral artery blood flow velocity, our group 92 previously found that older adults demonstrate a blunted cerebrovascular response (CVR) during an 
93

94

95

96

97

98

99

100

101

102

103

104

105

106

107

108

109

110

111

112

113

114

115

116

117

118

119

120

121

122

123

124

125

126

127

128

129

130

131

132

133

134

135

136

acute bout of moderate-intensity aerobic exercise (Sisante et al., 2019; Alwatban et al., 2020), which may serve as an early indicator of dysfunctional cerebrovascular regulation in preclinical older adult populations (Alwatban et al., 2020). Further, CVR was associated with elevated levels of A $\beta$ deposition, a hallmark of AD, in the brains of cognitively normal older adults (Sisante et al., 2019). Interestingly, cerebrovascular assessments performed at rest have not been able to discriminate cognitive status (Xie et al., 2016) or A $\beta$ levels (Sisante et al., 2019) in these preclinical older adult populations, suggesting that acute aerobic exercise assessment paradigms may enhance the detection of subtle impairments in vascular function in the early stages of disease. Consistent with this theory, dysfunctional regulation of cerebral blood flow has been detected in older adults with MCI (Xie et al., 2016) and precedes the onset of dementia, implicating its early mechanistic role in cognitive decline with aging (Iadecola, 2004). However, despite the apparent role of CVR dysfunction in the preclinical stages of brain pathology, the interactions between CVR and other key neurobiological factors linked to early-stage cognitive impairments and AD pathology remain unknown.

Possession of Apolipoprotein E4 (APOE4), the strongest known genetic risk factor for sporadic Alzheimer's disease (Heffernan et al., 2016), is strongly linked to cerebrovascular dysfunction (Montagne et al., 2020). Cognitively normal older adults with an APOE4 allele demonstrate a greater decline in cerebral blood flow with aging compared to noncarriers (Thambisetty et al., 2010), and an earlier blood-brain barrier breakdown that precipitates subsequent cognitive decline (Montagne et al., 2020). Recently, our group found that APOE4 carriers with lowest resting conductance in middle cerebral artery blood flow had the greatest $A \beta$ deposition, a relationship that was not present in noncarriers (Kaufman et al., 2021b). Yet, in the context of early-stage cognitive impairments, the behavioral relevance of APOE4 genotype in the presence of multifactorial aging pathologies among a heterogenous older adult population is poorly understood.

In this study, we aimed to 1) investigate the interactive effect of APOE4 carrier status on the relationship between CVR to exercise and $A \beta$ deposition and 2) test whether interactions between $A P O E 4$ carrier status, $\mathrm{CVR}$, and $\mathrm{A} \beta$ deposition affect cognitive performance involving response inhibition in a group of cognitively normal older adults. We hypothesized that 1) older adults with the most robust CVR to a bout of moderate-intensity aerobic exercise would have the lowest levels of $\mathrm{A} \beta$ deposition, with the strongest effect in APOE4 carriers, and 2) higher CVR would attenuate the negative effect of $A \beta$ deposition on response inhibition performance, particularly in APOE4 carriers.

\section{Materials and Methods}

\subsection{Participants}

We selected a subset of 70 older adults from a well-characterized data registry of 125 cognitively normal older adults from the University of Kansas Alzheimer's Disease Center (AHA 16GRNT30450008 (SB)) (Alwatban et al., 2020; Perdomo et al., 2020; Liu et al.). Our recruitment efforts within this cohort included those individuals with complete datasets for cerebrovascular function assessment, genetic $A P O E$ profiling, and structural neuroimaging for $A \beta$ deposition. Inclusion criteria were (1) between 65 and 90 years old, (2) normal cognition with the absence of clinical dementia or cognitive impairment and (3) physical ability to exercise. Exclusion criteria were (1) insulin-dependent diabetes; (2) peripheral neuropathy; (3) myocardial infarction or symptoms of coronary artery disease within 2 years; (4) congestive heart failure; (5) the presence of 1 or 2 APOE2 alleles. The experimental protocol was approved by the University of Kansas Institutional Review Board (IRB\#: STUDY00001444) and all participants provided written informed consent. 


\subsection{Acute bout of aerobic exercise on a recumbent stepper}

138 Participants underwent a bout of moderate-intensity aerobic exercise on a recumbent stepper in an experimental protocol previously described in detail (Billinger et al., 2017; Ward et al., 2018; Witte et al., 2019). Briefly, participants arrived to the laboratory between 7:30-9:00am after abstaining from caffeine for 12 hours, physical activity for 24 hours, and consuming a large meal for 2 hours. The exercise testing room was quiet, dimly lit, and temperature controlled between 22 and 24 degrees Celsius. Following 8 minutes of quiet rest seated on the recumbent stepper (NuStep T5XR), participants began an exercise familiarization bout to determine target power. Following an 8-minute seated rest recording, participants began exercising on the recumbent stepper, maintaining a step rate of 120 steps-per-minute and beginning at a resistance of $40 \mathrm{~W}$. The work resistance was increased until the participant reached the target heart rage range of 40-60\% of age-predicted heart rate reserve and maintained this target heart rate for one continuous minute (Billinger et al., 2017; Sisante et al., 2019). The participant then maintained exercise at this moderate-intensity hear rate zone for 8 minutes.

\subsection{Cerebro- and cardiovascular assessment and analyses}

During rest and exercise bouts, we measured left cerebral blood flow velocity using transcranial Doppler ultrasound (TCD) with a 2-MHz probe (RobotoC2MD, Multigon Industries) placed over the temporal window. If the left MCA signal was not obtainable, the right MCA was used. Data were acquired through an analog to digital data acquisition unit (NI-USB-6212, National Instruments) and custom written software operating in MATLAB (The Mathworks Inc). We used a 5-lead electrocardiogram for continuous heart rate monitoring. All data were sampled at $500 \mathrm{~Hz}$. Experimenters were blinded to $\mathrm{A} \beta$ deposition level, cognitive function, and $A P O E$ carrier status.

159 The mean MCA blood flow velocity was calculated during the 8-minute baseline rest and 8-minute moderate-intensity exercise bout. We calculated cerebrovascular response (CVR) as the difference between mean MCA blood flow velocity during exercise and mean MCA blood flow velocity at rest (Sisante et al., 2019).

\subsection{Structural neuroimaging of amyloid-beta (Aß) deposition}

Participants underwent Florbetapir PET scans on a GE Discovery ST-16 PET/CT scanner at approximately 50 minutes after administration of intravenous florbetapir 18F-AV45 (370 MBq). Continuous acquisition of two 5-minute PET brain frames were summed and attenuation corrected (Vidoni et al., 2021). The global standardized update value ratio (SUVR) was calculated using standard procedures described previously (Liu et al.) to determine global A $\beta$ deposition for each participant.

\subsection{APOE genotyping}

Whole blood was drawn and stored frozen at -80 degrees Celsius prior to genetic analyses using a Taqman single nucleotide polymorphism (SNP) allelic discrimination assay (ThermoFisher) to determine $A P O E$ genotype. Taqman probes were used to determine APOE4, APOE3, and APOE2 alleles to the two APOE-defining SNPs, rs429358 (C_3084793_20) and rs7412 (C_904973_10) (Kaufman et al., 2021c; Vidoni et al., 2021). Individuals were classified as APOE4 carrier in the presence of 1 or 2 APOE4 alleles (e.g. E3/E4, E4/E4). Individuals with homozygous E3 (e.g. E3/E3) were classified as a noncarriers. Because $A P O E 2$ is associated with reduced risk for Alzheimer's 
178 disease, all APOE2 carriers, whether homozygous or paired with a different APOE allele), were

179 excluded from analyses in the present study (Whitson et al., 2018; Kaufman et al., 2021a).

\subsection{Cognitive behavior assessment}

\subsubsection{Clinical neuropsychological test battery}

182 The Uniform Data Set (UDS) neuropsychological test battery and the Clinical Dementia Rating 183 (CDR) scale employed by the United States Alzheimer's Disease Research Center network (Morris, 184 1993; Monsell et al., 2016) were performed for all participants. All participants completed a standard in-person clinical and cognitive evaluation, during which the clinical CDR was performed by a trained clinician and the neuropsychological test battery by a trained psychometrist. Clinical and cognitive data were reviewed and finalized at a consensus diagnostic conference (Graves et al., 188 2015). All participants in the present analysis were rated as CDR $=0$ and cognitively normal.

189 Participant also completed a Mini-Mental State Exam (MMSE) (Folstein et al., 1975) (Table 1).

\subsubsection{Response Inhibition Performance}

191 Participants completed a Stroop test during which they were presented with three conditions: 1) Stroop word reading, where color words were printed in black ink, 2) Stroop color naming, in which rectangular color patches were shown, and 3) Stroop interference, in which color words were printed 194 in an incongruent ink color and participants were instructed to ignore the word and state the color of the ink. Each of the three conditions was presented individually in a 45 -second trial. The raw number of correct responses was recorded within the time limit for each condition.

The difference in the time for naming the colors in which the words are printed and the same colors printed in rectangles is the measure of the interference of conflicting word stimuli upon naming colors, standardized for speed differences between people (raw number of word interference on color /raw number of control color squares read) (Stroop, J, 1935).

201 We calculated response inhibition performance as the Stroop ratio, where:

$$
\text { Stroop Ratio }=\frac{\text { Stroop interference condition raw score }}{\text { Stroop color condition raw score }}
$$

A Stroop ratio $=1.0$ reflects perfect response inhibition performance and no interference of conflicting word stimuli. Here, the participant perfectly inhibited interfering word stimuli during the Stroop interference condition, with no slowing of response time relative to the control Stroop color condition. A Stroop ratio $=0.0$ reflects poor response inhibition performance and complete failure to inhibit conflicting word stimuli on a color naming task. Here, the participant was unable to state any 
210 We tested for normality and heterogeneity of variance of all data used for analyses using

211 Kolmogorov-Smirnov and Levene's tests, respectively. To test the effect of APOE4 carrier status on the relationship between CVR and $\mathrm{A} \beta$, we performed two-way moderated multiple linear regression analysis after controlling for participant age. To test for interactions between APOE4 carrier status, $\mathrm{CVR}$, and $\mathrm{A} \beta$ deposition on response inhibition performance, we performed a three-way multiple linear regression analyses after controlling for participant age. Specifically included in the three-way model were CVR, APOE4 carrier status, A $\beta$ deposition, and the interaction CVR $\times$ carrier status $\times$ $\mathrm{A} \beta$ deposition. The relationship between $\mathrm{A} \beta$ deposition and response inhibition performance was compared for participants across the range of CVR to aerobic exercise. To test response inhibition performance in APOE4 carriers and noncarriers as a function of CVR, we dichotomized participants by the group median CVR value and performed a two-way analysis of variance (ANOVA). All analyses were performed using SPSS version 25 with an a priori level of significance set to 0.05.

\section{Results}

Among the 70 total participants included in analyses (67\% female, 29\% APOE4 carriers, $71 \pm 5$ yo) (Table 1), there were no significant differences between APOE4 carriers and noncarriers in response inhibition performance $(p=.301)$, age $(p=.94)$, gender distribution $(p=.723)$, MMSE $(p=.916)$, or CVR to aerobic exercise $(p=.716)$ in carriers and noncarriers. APOE4 carriers had greater levels of A $\beta$ deposition compared to noncarriers $(p=.02)$.

228 Table 1. Participant characteristics.

229 MMSE = Mini-Mental State Exam; $A \beta=$ amyloid-beta; $C V R=$ cerebrovascular response to

\begin{tabular}{|c|c|c|c|}
\hline & $\begin{array}{c}\text { All } \\
(\mathrm{n}=70)\end{array}$ & $\begin{array}{c}\text { APOE4 (+) } \\
(\mathrm{n}=20)\end{array}$ & $\begin{array}{c}\text { APOE4 (-) } \\
(\mathrm{n}=50)\end{array}$ \\
\hline Age (years) & $\begin{array}{c}71 \pm 5 \\
{[65-87]}\end{array}$ & $\begin{array}{c}71 \pm 7 \\
{[65-87]}\end{array}$ & $71 \pm 5$ \\
& $\mathrm{~F}=47$ & $\mathrm{~F}=13$ & $\mathrm{~F}-81]$ \\
\hline Gender & $\mathrm{M}=23$ & $\mathrm{M}=7$ & $\mathrm{M}=16$ \\
\hline MMSE & $28.6 \pm 1.8$ & $28.6 \pm 1.4$ & $28.7 \pm 2.0$ \\
& {$[22.0-30.0]$} & {$[25.0-30.0]$} & {$[22.0-30.0]$} \\
\hline A $\beta$ & $1.09 \pm .17$ & $1.16 \pm .17$ & $1.07 \pm .17$ \\
Deposition & {$[0.86$ to 1.6$]$} & {$[0.98$ to 1.6$]$} & {$[0.86$ to 1.6$]$} \\
\hline CVR & $5.55 \pm 5.29$ & $5.18 \pm 4.58$ & $5.69 \pm 5.58$ \\
& {$[-13.00$ to 21.88$]$} & {$[-3.57$ to 13.78$]$} & {$[-13.00$ to 21.88$]$} \\
\hline Stroop & $0.52 \pm .12$ & $0.51 \pm .12$ & $0.53 \pm .17$ \\
ratio & {$[0.29$ to 1.0$]$} & {$[0.33$ to 0.82$]$} & {$[0.29$ to 1.00$]$} \\
\hline
\end{tabular}

$231 \mathrm{~A} \beta=$ amyloid-beta; $\mathrm{CVR}=$ cerebrovascular response to moderate-intensity aerobic exercise; [Range] 
After controlling for age, the two-way multiple regression model significantly predicted A $\beta$

234

235

236

237

238

239 deposition, $F_{4,69}=4.97$, p=0.001, $R^{2}=0.23$, adjusted $R^{2}=0.19$. Regression coefficients and standard errors are detailed in Table 2. A higher CVR predicted lower A $\beta$ deposition across all participants ( $p=0.013$ ) (Figure 1). Though APOE4 carriers showed a stronger relationship between CVR and A $\beta$ deposition $(\mathrm{r}=0.50)$ than noncarriers $(\mathrm{r}=0.38)$, the difference of this relationship was not significant ( $\mathrm{t}=-0.65, p=.517)$. There was a main effect of $A P O E$ carrier status on $\mathrm{A} \beta$ deposition in the model, with APOE4 carriers having greater A $\beta$ deposition than noncarriers $(p=.042)$ (Table 2).

Table 2. Two-way moderated multiple regression analysis testing the effect of APOE4 carrier status on the relationship between $\mathrm{CVR}$ and $\mathrm{A} \beta$ deposition after controlling for age, $\mathrm{N}=70$; Regression 242 model predicting $\mathrm{A} \beta$ deposition, $p=.0008$.

\begin{tabular}{|l|c|c|c|c|}
\hline Variable & $\boldsymbol{B}$ & $\mathrm{SE}_{B}$ & $\boldsymbol{\beta}$ & $\mathrm{p}$-value \\
\hline Intercept & 0.718 & -0.257 & & $.007^{*}$ \\
\hline Age & 0.005 & 0.004 & 0.152 & .176 \\
\hline CVR & -0.058 & 0.023 & -0.319 & $.013^{*}$ \\
\hline APOE4 carrier status (+) & 0.087 & 0.042 & 0.225 & $.042^{*}$ \\
\hline $\begin{array}{l}\text { Interaction APOE4 carrier } \\
\text { status (+) } \times \text { CVR }\end{array}$ & -0.032 & 0.049 & -0.080 & .517 \\
\hline
\end{tabular}

$\mathrm{A} \beta=$ amyloid-beta; $\mathrm{CVR}=$ cerebrovascular response to moderate-intensity aerobic exercise; $\mathrm{SE}_{B}=$ standard error of the coefficient; $B=$ unstandardized regression coefficient; $\beta=$ standardized coefficient; $* p<.05$

\subsection{Interactions between cerebrovascular function, amyloid-beta, and APOE genotype as predictors of response inhibition behavior}

The multiple regression model significantly predicted response inhibition performance, $F_{5,69}=3.55$, $p=.007, R^{2}=0.22$, adjusted $R^{2}=0.16$. Regression coefficients and standard errors are detailed in Table 3. There was a significant three-way interaction between $\mathrm{CVR}, A P O E$ carrier status, and A $\beta$ deposition ( $\mathrm{t}=2.79, p=.007)$, indicating differences in the predictive value of $\mathrm{A} \beta$ deposition and $\mathrm{CVR}$ on response inhibition performance for APOE4 carriers and noncarriers. Specifically, APOE4 carriers with higher CVR demonstrated better response inhibition performance $(p<.001)$, while noncarriers showed no significant effect of CVR ( $p=.112)$ (Figure 2). The model showed that APOE4 carrier status moderated the interactive effect of CVR and A $\beta$ deposition on response inhibition, as illustrated in Figure 3A \& B. APOE4 carriers who achieved a high CVR showed a positive relationship between $A \beta$ deposition and response inhibition, where carriers with a robust CVR to an acute bout of aerobic exercise performed better on the response inhibition task despite having high levels of A $\beta$ deposition. In contrast, APOE4 carriers who had lower CVR showed no relationship (Figure 3A). Noncarriers showed less effect of CVR on the relationship between AB deposition and response inhibition, where older adult noncarrier cognitive performance was independent of their CVR to the exercise bout (Figure 3B).

When the group was dichotomized by the group median CVR value $(5.7 \mathrm{~cm} / \mathrm{s})$, there was a significant $A P O E 4$ carrier group $\times$ CVR interaction $\left(\left(F_{3,69}\right)=12.84, p=.001\right)$ on response inhibition performance (Figure 3C). Post-hoc analyses revealed that APOE4 carriers with a high CVR ( $\geq$ $5.7 \mathrm{~cm} / \mathrm{s}$ ) demonstrated higher response inhibition performance compared to APOE4 carriers with low $\operatorname{CVR}(<5.7 \mathrm{~cm} / \mathrm{s})(t=-3.19, p=.005)$ and noncarriers with high CVR $(t=-2.51, p=.017)$. Among participants with low CVR, APOE4 carriers had poorer response inhibition performance compared to 
noncarriers $(t=2.64, p=.013)$. Within the noncarrier group, noncarriers with low CVR had higher response inhibition performance compared to those with high CVR $(t=2.18, p=.034)$ (Figure 3C).

Table 3. Three-way multiple regression analysis results to predict response inhibition performance after controlling for age, $\mathrm{N}=70$; Regression Model, $p=.007$.

\begin{tabular}{|l|c|c|c|c|}
\hline Variable & $\boldsymbol{B}$ & $\mathrm{SE}_{\boldsymbol{B}}$ & $\boldsymbol{\beta}$ & $\mathrm{p}$-value \\
\hline Intercept & 0.680 & 0.190 & & $.001^{*}$ \\
\hline Age & -0.006 & 0.002 & -0.258 & $.029^{*}$ \\
\hline $\mathrm{CVR}$ & -0.002 & 0.003 & -0.079 & .557 \\
\hline$A P O E 4$ carrier status (+) & -0.130 & 0.043 & -0.511 & $.004^{*}$ \\
\hline $\mathrm{A} \beta$ deposition & 0.236 & 0.084 & 0.355 & $.007^{*}$ \\
\hline $\begin{array}{l}\text { Interaction } A P O E 4 \text { carrier } \\
\text { status }(+){ }^{*} \mathrm{~A} \beta{ }^{*} \mathrm{CVR}\end{array}$ & 0.015 & 0.005 & 0.485 & $.007^{*}$ \\
\hline
\end{tabular}

$\mathrm{A} \beta=$ amyloid-beta; $\mathrm{CVR}=$ cerebrovascular response to moderate-intensity aerobic exercise; $\mathrm{SE}_{B}=$ standard error of the coefficient; $B=$ unstandardized regression coefficient; $\beta=$ standardized coefficient; $* p<.05$

\section{Discussion}

Findings of the present study shed light on the complex neurobiological interactions of factors known to influence the development of cognitive dysfunction with aging. Our results provide novel evidence for the behavioral significance of interactions between cerebrovascular function, individual genotype, and structural brain pathology. Despite having higher $\mathrm{A} \beta$ deposition, we found that APOE4 carriers who had a more robust CVR to exercise had higher response inhibition performance, an effect that was not present in noncarriers. These findings imply higher cerebrovascular function may serve a neuroprotective role for the preservation of cognitive executive function specifically in APOE4 carriers, possibly through increased resiliency to $A \beta$ deposition with aging. Building upon previous research, our findings support an individualized framework gleaned from a multifactorial approach for detection of subtle impairments in cognitive behavior prior to the onset of clinical syndrome associated with $\mathrm{AD}$ and related dementias. Together, these results may be informative for targeted precision-medicine approaches for early intervention and prevention of age-related declines in cognitive executive function.

\subsection{Cerebrovascular response (CVR) to exercise is linked to amyloid beta (A $\beta)$ deposition in the absence of clinical cognitive dysfunction}

Our findings support the assessment of CVR to acute aerobic exercise as a useful functional biomarker of structural brain pathology in the absence of clinically detectible impairments in cognitive function. In the present study, we found that cognitively normal older adults with blunted CVR to aerobic exercise demonstrated greater $A \beta$ deposition (Figure 1), a finding consistent with previous work from our laboratory (Sisante et al., 2019) across a larger cohort of older adults. This finding also supports previous research implicating cerebrovascular assessments performed under conditions of physiologic stress may be sensitive enough to detect the first signs of dysfunction in the regulation of cerebral blood flow that may be salient to subtle changes in cognitive function in 
302

303

304

305

306

307

308

309

310

311

312

313

314

315

316

317

318

319

320

321

322

323

324

325

326

327

328

329

330

331

332

333

334

335

336

337

338

339

340

341

342

343

344

345

346

347

with lower $\mathrm{A} \beta$ deposition in $A P O E 4$ carriers compared to noncarriers (i.e. steeper negative slope in APOE4 carriers (Figure 1)), the influence of APOE4 carrier status on the negative association between CVR and $A \beta$ deposition was not significant, in contrast to our a priori hypotheses (Table 2). This may be explained by the exclusion of older adults with MCI and a history of cardiovascular disease, which effectively narrowed the range of $C V R$ and $A \beta$ deposition in our participant cohort. In future studies that expand cognitive and cerebrovascular inclusion criteria, differences in this relationship between APOE4 carriers and noncarriers may emerge, in which the effect of negative association between CVR and $\mathrm{A} \beta$ deposition may be strongest in APOE4 carriers.

Our findings implicate common neurobiological mechanisms that contribute to cerebrovascular dysfunction and structural brain pathology in the early stages of brain aging. Our results demonstrate a negative relationship between CVR to exercise and $A \beta$ deposition that is consistent with a mechanistic link between $C V$ dysfunction and deposition of $A \beta$ in the aging brain. However, the causal nature of this relationship remains to be tested. For example, the presence of $A \beta$ may blunt the CVR to exercise through increased resistance of blood flow and perfusion to cortical tissues as blood flow velocity increases during aerobic exercise (Niwa et al., 2002; Iadecola, 2004). Conversely, poor cerebrovascular function may alter blood-brain barrier trafficking of $A \beta$, slowing the clearance of $A \beta$ and promoting its accumulation in the brain (Zlokovic et al., 2005). The later may be supported by evidence that habitual aerobic exercise appears to have no effect on $A \beta$ deposition in older adults (Vidoni et al., 2021), yet can improve cerebrovascular health (Thomas et al., 2013; Whitaker et al., 2020) and slow or, in some cases, reverse the development of cognitive impairment in older adults (Zhang et al., 2020). These observations may explain the neuroprotective effect of aerobic exercise against AD and related dementias (Tarumi and Zhang, 2018). Future research aimed at modulating each of these variables (e.g. aerobic exercise interventions that improve CVR) will provide insight into the directionality of the relationship between cerebrovascular function and structural brain pathology.

\subsection{Behavioral significance of interactions between individual genotype, cerebrovascular function, and brain structure}

Importantly, our findings provide evidence for the behavioral significance of interactions between individual genotype, cerebrovascular function, and brain structure. We found that cerebrovascular function interacts with individual $A P O E$ genotype and $A \beta$ deposition to influence an individual older adult's ability to inhibit a default and undesired response (Figure 2 and Figure 3A\&B). Our results are consistent with accumulating evidence within the field of aging neurobiology and the increasing recognition of the mixed pathology and multifactorial etiology that contributes to cognitive decline with aging (Iturria-Medina et al., 2016, 2017). Clinically, it is well known that an individual who has structural brain biomarker evidence of AD pathology (e.g. high levels of $\mathrm{A} \beta$ deposition) may paradoxically present with the absence of any behavioral indicator associated with AD clinical syndrome. Notably, vascular pathology and individual genotype (e.g. possession of the APOE4 allele) are frequently detected in the typical clinical presentation of AD (Iadecola, 2004; Xie et al., 2016; Ouellette and Lacoste, 2021), and can significantly increase an individual's risk for developing dementias (Heffernan et al., 2016). Building upon our current knowledge of each of these factors individually, our present results provide unique insight into interactions between these key neurobiological factors that can be gleaned from an interdisciplinary and multifactorial assessment of $\mathrm{AD}$ and related dementia risk factors. This rich multifactorial approach offers a powerful characterization of factors potentially influencing the early development of cognitive behavioral impairment that could have clinically meaningful implications for intervention, treatment, and prevention of $\mathrm{AD}$ and related dementia in preclinical older adult populations. 


\subsection{Role of cerebrovascular health as a function of individual genotype in the preservation of brain behavioral function with aging}

Our results suggest cerebrovascular function plays a key role in the early behavioral manifestations of cognitive executive dysfunction in older adults who carry the APOE4 allele. The three-way interaction model found that older adult $A P O E 4$ carriers with blunted CVR to exercise demonstrated poorer response inhibition performance, while CVR did not predict behavioral performance in noncarriers (Figure 2). The characterization of these interactions builds upon previous findings from our laboratory, in which we demonstrated measures poor cardiovascular health negatively influenced the CVR to exercise preferentially in APOE4 carriers (Kaufman et al., 2021c). Our findings are also consistent with previous evidence demonstrating a stronger effect of cardiovascular health on cognitive performance metrics in APOE4 carriers (Zade et al., 2010; Caselli et al., 2011; Shaaban et al., 2019). Specifically within a behavioral context, a key novel finding of the present study identifies the unique relationship between cerebrovascular regulation and response inhibition performance in APOE4 carriers compared to noncarriers. The positive relationship between CVR and response inhibition performance supports that higher cerebrovascular regulatory function may serve a unique neuroprotective function in early brain aging processes affecting cognition in APOE4 carriers, while playing less of a role in noncarriers. Interestingly, Kaufman et al. (2021a) revealed that cerebrovascular dysfunction in APOE4 carriers was differentially modifiable through an aerobic exercise intervention compared to noncarriers, with older adult APOE4 carriers showing greater improvements in cerebral blood flow following a year-long exercise intervention (Kaufman et al., 2021a). Together, these findings identify CVR to exercise as a useful early assessment tool in preclinical older adult populations and may provide a physiologically-informed target for precisionmedicine approaches aimed at preventing age-related declines in cognitive executive function.

\subsection{Genetic mechanisms influencing interactions between cerebrovascular function and structural brain pathology in the context of cognitive behavior}

Possession of an APOE4 allele may reveal unique genetic mechanisms that influence interactions between cerebrovascular regulation and structural brain pathology to influence cognitive behavior in older adults. Specifically, we found differential effects of CVR on the relationship between A $\beta$ and response inhibition in APOE4 carriers and noncarriers (Figure 3A\&B). In APOE4 carriers, individuals with a higher CVR and greater $A \beta$ deposition had higher response inhibition performance, an effect not observed in APOE4 carriers with low CVR or noncarriers (Figure 3A\&B). Further, APOE4 carriers with high CVR outperformed both carriers with low CVR and noncarriers on the response inhibition task (Figure 3C). In the face of age-related neuropathology, some individuals appear to utilize a neurologic "reserve" that enables behavioral compensation and attenuates cognitive decline (Stern et al., 2019). Findings in the present study provide preliminary evidence that the neuroprotective effect of high CVR in APOE4 carriers may act mechanistically through increased resilience to age-related structural pathology reflected in $A \beta$ deposition, potentially supporting a functional neurologic "reserve" in the aging brains of these individuals. We also found that, among participants with low CVR, APOE4 carriers had poorer response inhibition compared to noncarriers (Figure 3C). This suggests that cerebrovascular health not only serves a specialized protective effect to maintain cognitive function, but may additionally attenuate the rate of cognitive decline that is accelerated in APOE4 carriers (Caselli et al., 2004, 2007). It is conceivable that such differences in the resiliency effect of cerebrovascular function between APOE4 carriers and noncarriers may overlap with the neurobiological mechanisms that predispose APOE4 carriers to a higher genetic risk for the development of AD in the later stages of aging (Farrer et al., 1997; Heffernan et al., 2016). Further, our findings that CVR has a greater effect on cognitive function in 
394

395

396

397

398

399

400

401

402

403

404

405

406

407

408

409

410

411

412

413

414

415

416

417

418

419

420

421

422

423

424

425

426

427

428

429

430

431

432

433

434

435

436

437

438

439

APOE4 carriers compared to noncarriers supports the notion of different etiologies and pathologic disease processes leading to the development of $\mathrm{AD}$ for each $A P O E 4$ carriers and noncarriers (Emrani et al., 2020) , particularly that cerebrovascular dysfunction may play a preferential role in dementia pathogenesis for carriers (Høgh et al., 2001; Montagne et al., 2020). These preliminary findings motivate future research in a larger cohort of heterogeneous older adults that may additionally include $A P O E 2$ carriers, for whom the effect of resiliency to aging processes has been most consistently observed and reported (Farrer et al., 1997; James et al., 2017).

While high cerebrovascular function demonstrated a neuroprotective effect for APOE4 carriers, an interesting pattern in our data suggests that noncarriers may possess greater resiliency to potential negative effects of low CVR and high A $\beta$ deposition. While APOE4 carriers with high CVR had higher response inhibition performance, noncarriers with low CVR tended to have higher response inhibition performance than noncarriers with high CVR, though the relationship of this effect did not meet our a priori adopted level of significance (Figure 2) and showed a lower magnitude of effect compared to the contrasting effect observed in APOE4 carriers (Figure 3C). Previous studies found that, compared to cognitively normal APOE4 carriers, noncarriers showed differences in cortical neural function (Leuthold et al., 2013) and had greater adaptive processes to repeated trauma (James et al., 2017). Although the mechanisms are not yet fully understood, these interactive functional and structural processes appear to increase neuroprotection and promote brain resilience to specific disease processes in noncarriers, including the development of MCI (Pa et al., 2009), posttraumatic stress disorder (Peterson et al., 2015), and recovery from traumatic brain injury (Zhou et al., 2008). A greater ability to repair and protect against neuronal damage offered by the noncarrier-specific genotypic interaction (Mahley and Rall, 2000) may similarly increase noncarriers' resilience to the chronic neurotoxic effects poor cerebrovascular function on both the accumulation of $A \beta$ in the brain (Figure 1) and cognitive performance (Figure 2 and Figure 3) compared to APOE4 carriers in the present study. Notably, our exclusion of older adults with MCI would have biased the selection of only individuals with such genotypic resiliency (i.e. excluding those with low CVR and high A $\beta$ without this protective adaptive characteristic). The present participant selection criteria may explain poorer response inhibition performance in noncarriers with low A $\beta$ and high CVR (Figure 3B \& C). Future research in animal models may elucidate our mechanistic understanding of risk and resilience associated with individual $A P O E$ genotype and further identify epigenetic pathways underpinning individual-specific physiologic stress responses for neuronal repair and protection in the context of cerebrovascular health and cognitive dysfunction with aging.

\subsection{Cerebrovascular dysfunction affects neural networks involved in response inhibition in the early stages of cognitive dysfunction with aging}

Our hypothesis-driven analyses in the present study focused on the neurobiological factors influencing a highly specific aspect of cognition involving inhibition of a default and undesired response(Stroop, J, 1935; Hutchison et al., 2010). Yet, previous research supports the specificity of cerebrovascular function and individual APOE genotype (Whitson et al., 2018) effects on response inhibition behavior that engages inhibitory neural networks within the prefrontal cortex (PFC) (Wessel and Aron, 2017; Wessel et al., 2019). In neurologically-intact young adults, differences in acute cerebrovascular changes within the frontal cortical regions could be dissociated during a challenging Stroop task; here, participants with a greater frontal cortical hemodynamic response demonstrated higher response inhibition performance (Gratton et al., 2020). In aging populations, previous studies have demonstrated $\mathrm{n}$ that cortical inhibitory function is one of the earliest neural mechanisms affected by aging processes in cognitively normal older adults (Nielson et al., 2002; Heise et al., 2013; Levin et al., 2014; Rossiter et al., 2014; Legon et al., 2016), which may explain the 
presence of impaired response inhibition in older adults with absent clinical cognitive syndrome in the present study. Interestingly, PFC brain regions may preferentially benefit from the therapeutic effects of aerobic exercise interventions on cognition (Duchesne et al., 2015; Levin and Netz, 2015), supporting the robust interactive effect of cerebrovascular function on response inhibition performance in the present study. Taken together, these findings may inform the development of individualized approaches for therapeutic noninvasive brain stimulation (e.g. neuromodulation of PFC brain regions in preclinical APOE4 carriers) for the treatment and prevention of cognitive dysfunction with aging (Hsu et al., 2015).

\section{Conclusions}

449 Interactions between cerebrovascular function, individual genotype, and structural brain pathology may offer a useful and sensitive biomarker for early preclinical behavioral manifestations of cognitive impairment in older adults. Additionally, cerebrovascular response to aerobic exercise may provide a physiologically-informed target for precision-medicine approaches aimed at attenuating negative effects of structural brain pathology and preventing age-related declines in cognitive executive function. Future research may reveal that individuals with APOE4 carrier genotype show the highest therapeutic benefit for such intervention approaches.

\section{Conflict of Interest}

The authors declare that the research was conducted in the absence of any commercial or financial relationships that could be construed as a potential conflict of interest.

\section{$460 \quad$ Author Contributions}

JP conceived of the presented research question and designed the analysis for this study. JB and SB provided oversight to the data collected and supervised all data analyses. CK contributed to recruitment and data collection. JP constructed the figures and the first draft of this manuscript. EV oversight and interpretation for the individual genotyping. SB supervised the findings of this work. All authors discussed the results and contributed to the final manuscript.

\section{Funding}

Research reported in this publication was supported by the Eunice Kennedy Shriver National Institute of Child Health \& Human Development and the National Institute on Aging of the National Institutes of Health [NIH F32HD096816 (JP), P30 AG072973, P30AG035982, R01 AG043962, UL1TR000001], the American Heart Association [16GRNT30450008 (SB)], and the Georgia Holland Endowment Fund. Gifts from Frank and Evangeline Thompson (JMB), The Ann and Gary Dickinson Family Charitable Foundation, John and Marny Sherman, Brad and Libby Bergman supported amyloid measurement infrastructure. Lilly Pharmaceuticals provided a grant to support F18-AV45 doses and partial scan costs. The content of this publication is solely the responsibility of the authors and does not necessarily represent the official views of the National Institutes of Health or any other funding agency. 


\section{References}

480

Alwatban, M. R., Liu, Y., Perdomo, S. J., Ward, J. L., Vidoni, E. D., Burns, J. M., et al. (2020). TCD Cerebral Hemodynamic Changes during Moderate-Intensity Exercise in Older Adults. Journal of Neuroimaging 30, 76-81. doi:10.1111/jon.12675.

Billinger, S. A., Craig, J. C., Kwapiszeski, S. J., Sisante, J.-F. V., Vidoni, E. D., Maletsky, R., et al. (2017). Dynamics of middle cerebral artery blood flow velocity during moderate-intensity exercise. Journal of Applied Physiology 122, 1125-1133. doi:10.1152/japplphysiol.00995.2016.

Bracko, O., Hernández, J. C. C., Park, L., Nishimura, N., and Schaffer, C. B. (2021). Causes and consequences of baseline cerebral blood flow reductions in Alzheimer's disease: Journal of Cerebral Blood Flow \& Metabolism. doi:10.1177/0271678X20982383.

Bundo, M., Inao, S., Nakamura, A., Kato, T., Ito, K., Tadokoro, M., et al. (2002). Changes of neural activity correlate with the severity of cortical ischemia in patients with unilateral major cerebral artery occlusion. Stroke 33, 61-66. doi:10.1161/hs0102.101816.

Caselli, R. J., Dueck, A. C., Locke, D. E. C., Sabbagh, M. N., Ahern, G. L., Rapcsak, S. Z., et al. (2011). Cerebrovascular risk factors and preclinical memory decline in healthy APOE $\varepsilon 4$ homozygotes. Neurology 76, 1078-1084. doi:10.1212/WNL.0b013e318211c3ae.

Caselli, R. J., Reiman, E. M., Locke, D. E. C., Hutton, M. L., Hentz, J. G., Hoffman-Snyder, C., et al. (2007). Cognitive domain decline in healthy apolipoprotein E epsilon4 homozygotes before the diagnosis of mild cognitive impairment. Arch Neurol 64, 1306-1311. doi:10.1001/archneur.64.9.1306.

Caselli, R. J., Reiman, E. M., Osborne, D., Hentz, J. G., Baxter, L. C., Hernandez, J. L., et al. (2004). Longitudinal changes in cognition and behavior in asymptomatic carriers of the APOE e4 allele. Neurology 62, 1990-1995. doi:10.1212/01.wnl.0000129533.26544.bf.

Coelho, F. G. de M., Stella, F., de Andrade, L. P., Barbieri, F. A., Santos-Galduróz, R. F., Gobbi, S., et al. (2012). Gait and risk of falls associated with frontal cognitive functions at different stages of Alzheimer's disease. Neuropsychol Dev Cogn B Aging Neuropsychol Cogn 19, 644656. doi:10.1080/13825585.2012.661398.

Duchesne, C., Lungu, O., Nadeau, A., Robillard, M. E., Boré, A., Bobeuf, F., et al. (2015). Enhancing both motor and cognitive functioning in Parkinson's disease: Aerobic exercise as a rehabilitative intervention. Brain Cogn 99, 68-77. doi:10.1016/j.bandc.2015.07.005.

Emrani, S., Arain, H. A., DeMarshall, C., and Nuriel, T. (2020). APOE4 is associated with cognitive and pathological heterogeneity in patients with Alzheimer's disease: a systematic review. Alzheimers Res Ther 12, 141. doi:10.1186/s13195-020-00712-4.

Farrer, L. A., Cupples, L. A., Haines, J. L., Hyman, B., Kukull, W. A., Mayeux, R., et al. (1997). Effects of age, sex, and ethnicity on the association between apolipoprotein E genotype and Alzheimer disease. A meta-analysis. APOE and Alzheimer Disease Meta Analysis Consortium. JAMA 278, 1349-1356. 
517

518

519

520

521

522

523

524

525

526

527

528

529

530

531

532

533

534

535

536

537

538

539

540

541

542

543

544

545

546

547

548

549

550

551

552

Ferguson, B. (2014). ACSM's Guidelines for Exercise Testing and Prescription 9th Ed. 2014. J Can Chiropr Assoc 58, 328.

Folstein, M. F., Folstein, S. E., and McHugh, P. R. (1975). "Mini-mental state". A practical method for grading the cognitive state of patients for the clinician. J Psychiatr Res 12, 189-198. doi:10.1016/0022-3956(75)90026-6.

Gratton, G., Weaver, S. R., Burley, C. V., Low, K. A., Maclin, E. L., Johns, P. W., et al. (2020). Dietary flavanols improve cerebral cortical oxygenation and cognition in healthy adults. Sci Rep 10, 19409. doi:10.1038/s41598-020-76160-9.

Graves, R. S., Mahnken, J. D., Swerdlow, R. H., Burns, J. M., Price, C., Amstein, B., et al. (2015). Open-source, Rapid Reporting of Dementia Evaluations. J Registry Manag 42, 111-114.

Hampel, H. (2013). Amyloid- $\beta$ and cognition in aging and Alzheimer's disease: molecular and neurophysiological mechanisms. J Alzheimers Dis 33 Suppl 1, S79-86. doi:10.3233/JAD2012-129003.

Heffernan, A. L., Chidgey, C., Peng, P., Masters, C. L., and Roberts, B. R. (2016). The Neurobiology and Age-Related Prevalence of the $\varepsilon 4$ Allele of Apolipoprotein E in Alzheimer's Disease Cohorts. J Mol Neurosci 60, 316-324. doi:10.1007/s12031-016-0804-x.

Heise, K.-F., Zimerman, M., Hoppe, J., Gerloff, C., Wegscheider, K., and Hummel, F. C. (2013). The Aging Motor System as a Model for Plastic Changes of GABA-Mediated Intracortical Inhibition and Their Behavioral Relevance. J. Neurosci. 33, 9039-9049. doi:10.1523/JNEUROSCI.4094-12.2013.

Høgh, P., Knudsen, G. M., Kjaer, K. H., Jørgensen, O. S., Paulson, O. B., and Waldemar, G. (2001). Single photon emission computed tomography and apolipoprotein $\mathrm{E}$ in Alzheimer's disease: impact of the epsilon4 allele on regional cerebral blood flow. J Geriatr Psychiatry Neurol 14, 42-51. doi:10.1177/089198870101400110.

Hsu, W.-Y., Ku, Y., Zanto, T. P., and Gazzaley, A. (2015). Effects of noninvasive brain stimulation on cognitive function in healthy aging and Alzheimer's disease: a systematic review and meta-analysis. Neurobiol Aging 36, 2348-2359. doi:10.1016/j.neurobiolaging.2015.04.016.

Hutchison, K. A., Balota, D. A., and Duchek, J. M. (2010). The Utility of Stroop Task Switching as a Marker for Early Stage Alzheimer's Disease. Psychol Aging 25, 545-559. doi:10.1037/a0018498.

Iadecola, C. (2004). Neurovascular regulation in the normal brain and in Alzheimer's disease. Nat Rev Neurosci 5, 347-360. doi:10.1038/nrn1387.

Iturria-Medina, Y., Carbonell, F. M., Sotero, R. C., Chouinard-Decorte, F., Evans, A. C., and Alzheimer's Disease Neuroimaging Initiative (2017). Multifactorial causal model of brain (dis)organization and therapeutic intervention: Application to Alzheimer's disease. Neuroimage 152, 60-77. doi:10.1016/j.neuroimage.2017.02.058. 
553

554

555

556

557

558

559

560

561

562

563

564

565

566

567

568

569

570

571

572

573

574

575

576

577

578

579

580

581

582

583

584

585

586

587

588

589

590

591

Iturria-Medina, Y., Sotero, R. C., Toussaint, P. J., Mateos-Pérez, J. M., and Evans, A. C. (2016). Early role of vascular dysregulation on late-onset Alzheimer's disease based on multifactorial data-driven analysis. Nature Communications 7, 11934. doi:10.1038/ncomms11934.

Jack, C. R., Wiste, H. J., Vemuri, P., Weigand, S. D., Senjem, M. L., Zeng, G., et al. (2010). Brain beta-amyloid measures and magnetic resonance imaging atrophy both predict time-toprogression from mild cognitive impairment to Alzheimer's disease. Brain 133, 3336-3348. doi:10.1093/brain/awq277.

James, L. M., Engdahl, B. E., and Georgopoulos, A. P. (2017). Apolipoprotein E: the resilience gene. Exp Brain Res 235, 1853-1859. doi:10.1007/s00221-017-4941-4.

Kaufman, C. S., Honea, R. A., Pleen, J., Lepping, R. J., Watts, A., Morris, J. K., et al. (2021a). Aerobic exercise improves hippocampal blood flow for hypertensive Apolipoprotein E4 carriers. J Cereb Blood Flow Metab 41, 2026-2037. doi:10.1177/0271678X21990342.

Kaufman, C. S., Morris, J. K., Vidoni, E. D., Burns, J. M., and Billinger, S. A. (2021b). Apolipoprotein E4 moderates the association between vascular risk factors \& brain pathology. Journal of Alzheimer Disease \& Associated Disorders. doi:epub ahead of print.

Kaufman, C. S., Morris, J. K., Vidoni, E. D., Burns, J. M., and Billinger, S. A. (2021c). Apolipoprotein E4 Moderates the Association Between Vascular Risk Factors and Brain Pathology. Alzheimer Dis Assoc Disord. doi:10.1097/WAD.0000000000000442.

Kirova, A.-M., Bays, R. B., and Lagalwar, S. (2015). Working memory and executive function decline across normal aging, mild cognitive impairment, and Alzheimer's disease. Biomed Res Int 2015, 748212. doi:10.1155/2015/748212.

Legon, W., Punzell, S., Dowlati, E., Adams, S. E., Stiles, A. B., and Moran, R. J. (2016). Altered Prefrontal Excitation/Inhibition Balance and Prefrontal Output: Markers of Aging in Human Memory Networks. Cereb Cortex 26, 4315-4326. doi:10.1093/cercor/bhv200.

Leuthold, A. C., Mahan, M. Y., Stanwyck, J. J., Georgopoulos, A., and Georgopoulos, A. P. (2013). The number of cysteine residues per mole in apolipoprotein $\mathrm{E}$ affects systematically synchronous neural interactions in women's healthy brains. Exp Brain Res 226, 525-536. doi:10.1007/s00221-013-3464-x.

Levin, O., Fujiyama, H., Boisgontier, M. P., Swinnen, S. P., and Summers, J. J. (2014). Aging and motor inhibition: a converging perspective provided by brain stimulation and imaging approaches. Neuroscience and Biobehavioral Reviews 43, 100-117. doi:10.1016/j.neubiorev.2014.04.001.

Levin, O., and Netz, Y. (2015). Aerobic training as a means to enhance inhibition: what's yet to be studied? European Review of Aging and Physical Activity: Official Journal of the European Group for Research into Elderly and Physical Activity 12, 14. doi:10.1186/s11556-015-01609.

Liu, Y., Perdomo, S. J., Ward, J., Vidoni, E. D., Sisante, J. F., Kirkendoll, K., et al. Vascular Health is Associated with Amyloid- $\beta$ in Cognitively Normal Older Adults. J Alzheimers Dis 70, 467-475. doi:10.3233/JAD-181268. 
592

593

594

595

596

597

598

599

600

601

602

603

604

605

606

607

608

609

610

611

612

613

614

615

616

617

618

619

620

621

622

623

624

625

626

627

Lorius, N., Locascio, J. J., Rentz, D. M., Johnson, K. A., Sperling, R. A., Viswanathan, A., et al. (2015). Vascular disease and risk factors are associated with cognitive decline in the Alzheimer's disease spectrum. Alzheimer Dis Assoc Disord 29, 18-25. doi:10.1097/WAD.0000000000000043.

Mahley, R. W., and Rall, S. C. (2000). Apolipoprotein E: far more than a lipid transport protein. Annu Rev Genomics Hum Genet 1, 507-537. doi:10.1146/annurev.genom.1.1.507.

Monsell, S. E., Dodge, H. H., Zhou, X.-H., Bu, Y., Besser, L. M., Mock, C., et al. (2016). Results From the NACC Uniform Data Set Neuropsychological Battery Crosswalk Study. Alzheimer Dis Assoc Disord 30, 134-139. doi:10.1097/WAD.0000000000000111.

Montagne, A., Nation, D. A., Sagare, A. P., Barisano, G., Sweeney, M. D., Chakhoyan, A., et al. (2020). APOE4 leads to blood-brain barrier dysfunction predicting cognitive decline. Nature 581, 71-76. doi:10.1038/s41586-020-2247-3.

Morris, J. C. (1993). The Clinical Dementia Rating (CDR): current version and scoring rules. Neurology 43, 2412-2414. doi:10.1212/wnl.43.11.2412-a.

Nielson, K. A., Langenecker, S. A., and Garavan, H. (2002). Differences in the functional neuroanatomy of inhibitory control across the adult life span. Psychology and Aging 17, 5671. doi:10.1037//0882-7974.17.1.56.

Niwa, K., Kazama, K., Younkin, L., Younkin, S. G., Carlson, G. A., and Iadecola, C. (2002). Cerebrovascular autoregulation is profoundly impaired in mice overexpressing amyloid precursor protein. Am J Physiol Heart Circ Physiol 283, H315-323. doi:10.1152/ajpheart.00022.2002.

Ogoh, S., and Ainslie, P. N. (2009a). Cerebral blood flow during exercise: mechanisms of regulation. J Appl Physiol (1985) 107, 1370-1380. doi:10.1152/japplphysiol.00573.2009.

Ogoh, S., and Ainslie, P. N. (2009b). Regulatory mechanisms of cerebral blood flow during exercise: new concepts. Exerc Sport Sci Rev 37, 123-129. doi:10.1097/JES.0b013e3181aa64d7.

Ogoh, S., Sato, K., Okazaki, K., Miyamoto, T., Hirasawa, A., Morimoto, K., et al. (2013). Blood flow distribution during heat stress: cerebral and systemic blood flow. J Cereb Blood Flow Metab 33, 1915-1920. doi:10.1038/jcbfm.2013.149.

Ouellette, J., and Lacoste, B. (2021). From Neurodevelopmental to Neurodegenerative Disorders: The Vascular Continuum. Front Aging Neurosci 13, 749026. doi:10.3389/fnagi.2021.749026.

Pa, J., Boxer, A., Chao, L. L., Gazzaley, A., Freeman, K., Kramer, J., et al. (2009). Clinicalneuroimaging characteristics of dysexecutive mild cognitive impairment. Ann Neurol 65, 414-423. doi:10.1002/ana.21591.

Palmer, J. A., Payne, A. M., Ting, L. H., and Borich, M. R. (2021). Cortical engagement metrics during reactive balance are associated with distinct aspects of balance behavior in older adults. Front. Aging Neurosci. 13. doi:10.3389/fnagi.2021.684743. 
628

629

630

631

632

633

634

635

636

637

638

639

640

641

642

643

644

645

646

647

648

649

650

651

652

653

654

655

656

657

658

659

660

661

662

663

664

665

Perdomo, S. J., Ward, J., Liu, Y., Vidoni, E. D., Sisante, J. F., Kirkendoll, K., et al. (2020). Cardiovascular disease risk is associated with middle cerebral artery blood flow velocity in older adults. Cardiopulm Phys Ther J 31, 38-46. doi:10.1097/cpt.0000000000000110.

Peterson, C. K., James, L. M., Anders, S. L., Engdahl, B. E., and Georgopoulos, A. P. (2015). The Number of Cysteine Residues per Mole in Apolipoprotein E Is Associated With the Severity of PTSD Re-Experiencing Symptoms. J Neuropsychiatry Clin Neurosci 27, 157-161. doi:10.1176/appi.neuropsych.13090205.

Rossiter, H. E., Davis, E. M., Clark, E. V., Boudrias, M.-H., and Ward, N. S. (2014). Beta oscillations reflect changes in motor cortex inhibition in healthy ageing. Neurolmage 91 , 360-365. doi:10.1016/j.neuroimage.2014.01.012.

Sato, K., Ogoh, S., Hirasawa, A., Oue, A., and Sadamoto, T. (2011). The distribution of blood flow in the carotid and vertebral arteries during dynamic exercise in humans. J Physiol 589, 28472856. doi:10.1113/jphysiol.2010.204461.

Shaaban, C. E., Jia, Y., Chang, C.-C. H., and Ganguli, M. (2019). Independent and joint effects of vascular and cardiometabolic risk factor pairs for risk of all-cause dementia: a prospective population-based study. Int Psychogeriatr 31, 1421-1432. doi:10.1017/S1041610219001066.

Sisante, J.-F. V., Vidoni, E. D., Kirkendoll, K., Ward, J., Liu, Y., Kwapiszeski, S., et al. (2019). Blunted cerebrovascular response is associated with elevated beta-amyloid. J Cereb Blood Flow Metab 39, 89-96. doi:10.1177/0271678X17732449.

Smith, K. J., and Ainslie, P. N. (2017). Regulation of cerebral blood flow and metabolism during exercise. Experimental Physiology 102, 1356-1371. doi:10.1113/EP086249.

Solis, E., Hascup, K. N., and Hascup, E. R. (2020). Alzheimer's Disease: The Link Between Amyloid- $\beta$ and Neurovascular Dysfunction. J Alzheimers Dis 76, 1179-1198. doi:10.3233/JAD-200473.

Steinback, C. D., and Poulin, M. J. (2016). Influence of Hypoxia on Cerebral Blood Flow Regulation in Humans. Adv Exp Med Biol 903, 131-144. doi:10.1007/978-1-4899-7678-9_9.

Stern, Y., Barnes, C. A., Grady, C., Jones, R. N., and Raz, N. (2019). Brain reserve, cognitive reserve, compensation, and maintenance: operationalization, validity, and mechanisms of cognitive resilience. Neurobiology of Aging 83, 124-129. doi:10.1016/j.neurobiolaging.2019.03.022.

Stroop, J (1935). Studies of interference in serial verbal reactions. J Exp Psychol, 643-62. doi:doi.org/10.1037/h0054651.

Sweeney, M. D., Kisler, K., Montagne, A., Toga, A. W., and Zlokovic, B. V. (2018). The role of brain vasculature in neurodegenerative disorders. Nature Neuroscience 21, 1318-1331. doi:10.1038/s41593-018-0234-X.

Tarumi, T., and Zhang, R. (2018). Cerebral blood flow in normal aging adults: cardiovascular determinants, clinical implications, and aerobic fitness. Journal of Neurochemistry 144, 595608. doi:https://doi.org/10.1111/jnc.14234. 
666

667

668

669

670

671

672

673

674

675

676

677

678

679

680

681

682

683

684

685

686

687

688

689

690

691

692

693

694

695

696

697

698

699

700

701

702

703

704

Thambisetty, M., Beason-Held, L., An, Y., Kraut, M. A., and Resnick, S. M. (2010). APOE epsilon4 genotype and longitudinal changes in cerebral blood flow in normal aging. Arch Neurol 67, 93-98. doi:10.1001/archneurol.2009.913.

Thomas, B. P., Yezhuvath, U. S., Tseng, B. Y., Liu, P., Levine, B. D., Zhang, R., et al. (2013). Lifelong aerobic exercise preserved baseline cerebral blood flow but reduced vascular reactivity to CO2. Journal of magnetic resonance imaging: JMRI 38, 1177-1183. doi:10.1002/jmri.24090.

Vidoni, E. D., Morris, J. K., Watts, A., Perry, M., Clutton, J., Sciver, A. V., et al. (2021). Effect of aerobic exercise on amyloid accumulation in preclinical Alzheimer's: A 1-year randomized controlled trial. PLOS ONE 16, e0244893. doi:10.1371/journal.pone.0244893.

Ward, J. L., Craig, J. C., Liu, Y., Vidoni, E. D., Maletsky, R., Poole, D. C., et al. (2018). Effect of healthy aging and sex on middle cerebral artery blood velocity dynamics during moderateintensity exercise. Am J Physiol Heart Circ Physiol 315, H492-H501. doi:10.1152/ajpheart.00129.2018.

Wessel, J. R., and Aron, A. R. (2017). On the Globality of Motor Suppression: Unexpected Events and Their Influence on Behavior and Cognition. Neuron 93, 259-280. doi:10.1016/j.neuron.2016.12.013.

Wessel, J. R., Waller, D. A., and Greenlee, J. D. (2019). Non-selective inhibition of inappropriate motor-tendencies during response-conflict by a fronto-subthalamic mechanism. eLife 8. doi:10.7554/eLife.42959.

Whitaker, A. A., Alwatban, M., Freemyer, A., Perales-Puchalt, J., and Billinger, S. A. (2020). Effects of high intensity interval exercise on cerebrovascular function: A systematic review. PLOS ONE 15, e0241248. doi:10.1371/journal.pone.0241248.

Whitson, H. E., Potter, G. G., Feld, J. A., Plassman, B. L., Reynolds, K., Sloane, R., et al. (2018). Dual-Task Gait and Alzheimer's Disease Genetic Risk in Cognitively Normal Adults: A Pilot Study. J Alzheimers Dis 64, 1137-1148. doi:10.3233/JAD-180016.

Witte, E., Liu, Y., Ward, J. L., Kempf, K. S., Whitaker, A., Vidoni, E. D., et al. (2019). Exercise Intensity and Middle Cerebral Artery Dynamics in Humans. Respir Physiol Neurobiol 262, 32-39. doi:10.1016/j.resp.2019.01.013.

Wolters Frank J., Zonneveld Hazel I., Hofman Albert, van der Lugt Aad, Koudstaal Peter J., Vernooij Meike W., et al. (2017). Cerebral Perfusion and the Risk of Dementia. Circulation 136, 719-728. doi:10.1161/CIRCULATIONAHA.117.027448.

Xie, L., Dolui, S., Das, S. R., Stockbower, G. E., Daffner, M., Rao, H., et al. (2016). A brain stress test: Cerebral perfusion during memory encoding in mild cognitive impairment. Neuroimage Clin 11, 388-397. doi:10.1016/j.nicl.2016.03.002.

Zade, D., Beiser, A., McGlinchey, R., Au, R., Seshadri, S., Palumbo, C., et al. (2010). Interactive Effects of Apolipoprotein E Type 4 Genotype and Cerebrovascular Risk on Neuropsychological Performance and Structural Brain Changes. Journal of Stroke and Cerebrovascular Diseases 19, 261-268. doi:10.1016/j.jstrokecerebrovasdis.2009.05.001. 
705

706

707

708

709

710

711

712

713

714

715

716

717

718

719

720

721

722

723

724

725

726

727

728

729

730

731

732

733

734

735

736

737

738

739

740

741

742

743

744

745

Zhang, D., Lu, Y., Zhao, X., Zhang, Q., and Li, L. (2020). Aerobic Exercise Attenuates Neurodegeneration and Promotes Functional Recovery - Why It Matters For Neurorehabilitation \& Neural Repair. Neurochemistry International, 104862. doi:10.1016/j.neuint.2020.104862.

Zhou, W., Xu, D., Peng, X., Zhang, Q., Jia, J., and Crutcher, K. A. (2008). Meta-analysis of APOE4 allele and outcome after traumatic brain injury. J Neurotrauma 25, 279-290. doi:10.1089/neu.2007.0489.

Zlokovic, B. V., Deane, R., Sallstrom, J., Chow, N., and Miano, J. M. (2005). Neurovascular pathways and Alzheimer amyloid beta-peptide. Brain Pathol 15, 78-83. doi:10.1111/j.17503639.2005.tb00103.x.

\section{Data Availability Statement}

The datasets generated and analyzed supporting the conclusions of this article will be made available by the authors upon request, without undue reservation.

\section{Captions to Figures}

Figure 1. Relationship between cerebrovascular response to moderate intensity exercise and global amyloid-beta $(\mathrm{A} \beta)$ burden. A higher CVR predicted lower $A \beta$ deposition across all participants $(p=.013)$.

Figure 2. Association between cerebrovascular response to moderate-intensity aerobic exercise and response inhibition performance predicted from the regression model in APOE4 carriers $(\mathrm{n}=20)$ (solid line) and noncarriers $(\mathrm{n}=50)$ (broken line). In APOE4 carriers, greater cerebrovascular response to exercise was associated with better performance on the Stroop response inhibition task $(p<.001)$. In noncarriers, no relationship was observed $(p=.112)$.

Results for this regression model are detailed in Table 3.

Figure 3. Association between $A \beta$ deposition and response inhibition performance illustrated as a function of cerebrovascular response (CVR) to moderate-intensity aerobic exercise in APOE4 carriers (black color) $(\mathrm{n}=20)(\mathbf{A})$ and noncarriers (grey color) $(\mathrm{n}=50)(\mathbf{B})$. APOE4 carriers with high CVR $(\geq 5.7 \mathrm{~cm} / \mathrm{s}$ ) (solid line) showed a positive relationship between $A \beta$ deposition and response inhibition performance, while APOE4 carriers with low CVR $(<5.7 \mathrm{~cm} / \mathrm{s})($ broken line) showed no positive effect. CVR also showed a lower effect on response inhibition in noncarriers. Results for this regression model are detailed in Table 3. (C) Response inhibition performance in APOE4 carriers and noncarriers with low and high CVR. There was a significant $A P O E 4$ carrier group $\times$ CVR interaction. APOE4 carriers with high CVR had higher response inhibition performance compared to APOE4 carriers with low CVR $(p=.005)$ and noncarriers ( $\mathrm{p}=.017)$. Among individuals with low CVR, APOE4 carriers had lower response inhibition performance compared to noncarriers $(p=.013)$. Within the noncarrier group, noncarriers with low CVR had higher response inhibition performance compared to those with high CVR $(p=.034) .{ }^{*} p<.05, * * p<.01$.

CVR is illustrated as a median $(5.7 \mathrm{~cm} / \mathrm{s})$ split of the group, where High CVR $\geq 5.7 \mathrm{~cm} / \mathrm{s}$; Low CVR < $5.7 \mathrm{~cm} / \mathrm{s}$. 


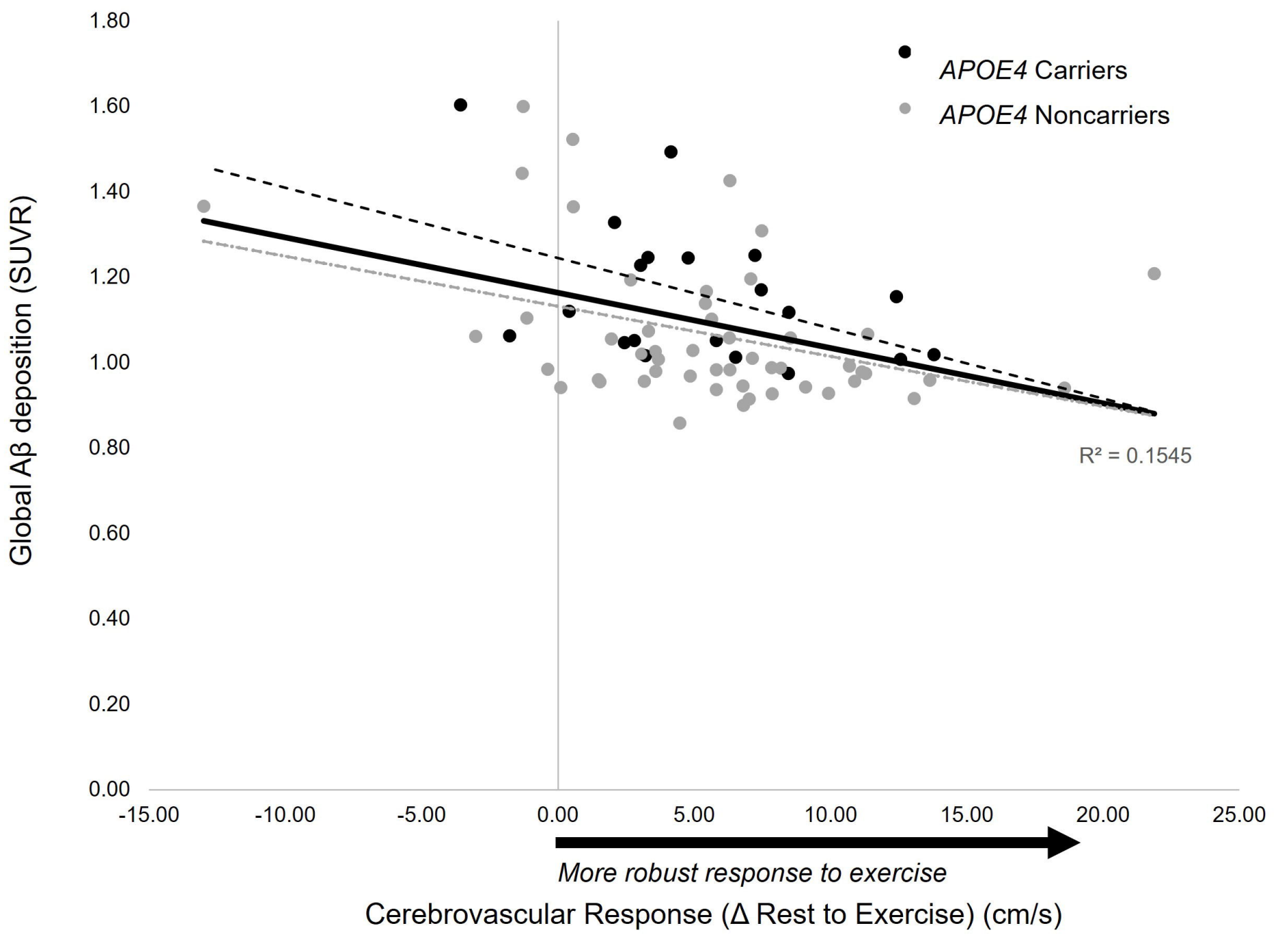




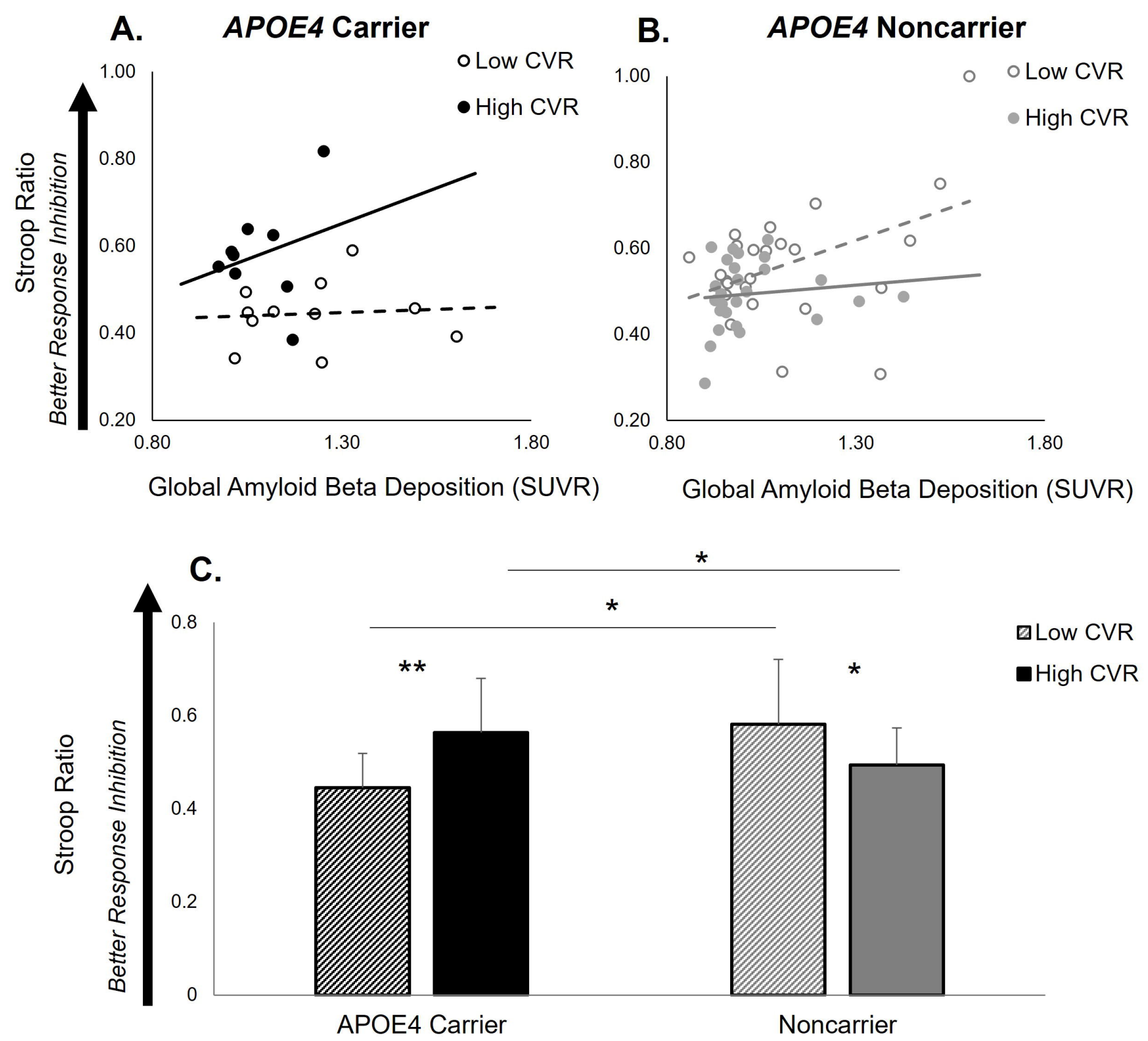

